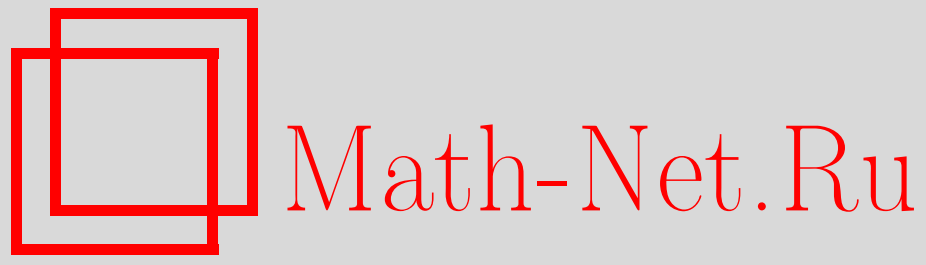

А. В. Лебедев, Топологически свободные частичные действия и точные представления скрещенных произведений, Функи. анализ и его прил., 2005, том 39, выпуск 3, 54-63

DOI: https://doi.org/10.4213/faa74

Использование Общероссийского математического портала Math-Net.Ru подразумевает, что вы прочитали и согласны с пользовательским соглашением http://www. mathnet.ru/rus/agreement

Параметры загрузки:

IP : 54.197 .130 .99

26 апреля 2023 г., 02:36:55 


\title{
Топологически свободные частичные действия и точные представления скрещенных произведений
}

\author{
(C) 2005. А. В. ЛЕБЕДЕВ
}

\section{§1. Введение}

Понятие скрещенного произведения $C^{*}$-алгебры на частичное действие группы $\mathbb{Z}$ частичными автоморфизмами введено Экселем [1]. Оно было распространено Мак-Кланаханом [2] на случай частичных действий дискретных групп. В последнее время скрещенные произведения находят все новые приложения как в анализе, так и в других разделах математики и математической физики. Интересное обсуждение этих и связанных с ними объектов можно найти, например, в [3]. Для исследования универсальных объектов такого типа важно иметь информацию об их точных представлениях. Описание характеристических свойств этих представлений и является темой данной статьи. Одними из основных условий, при выполнении которых можно получить такие представления, являются существование не увеличивающего норму условного ожидания на «алгебру коэффициентов» (свойство $(*)$, см. §2) и топологическая свобода частичного действия. В работе показано, что топологическая свобода частичного действия влечет за собой свойство $(*)$.

В этом вводном параграфе приведены необходимые сведения о частичных действиях и соответствующих скрещенных произведениях. В следующем параграфе вводится понятие топологически свободного частичного действия и доказывается основной результат статьи (теорема 2.7), связывающий топологическую свободу действия и свойство $(*)$. Далее, в 33 , на основе этих результатов мы описываем условия существования точных представлений скрещенных произведений и приведенных скрещенных произведений.

Пусть $A$ есть $C^{*}$-алгебра и $G$ - дискретная группа.

ОПРЕДЕЛЕНИЕ. Частичным действием группы $G$ на $A$ называется набор $\alpha=\left\{\alpha_{g}\right\}_{g \in G}$ изоморфизмов $\alpha_{g}: D_{g^{-1}} \rightarrow D_{g}$ замкнутых двусторонних *-идеалов $C^{*}$-алгебры $A$, для которых

(1) $\alpha_{g}\left(D_{g^{-1}} \cap D_{h}\right) \subset D_{g h}, g, h \in G$;

(2) $\alpha_{h g}(d)=\alpha_{h}\left(\alpha_{g}(d)\right), d \in D_{g^{-1}} \cap D_{g^{-1} h^{-1}}$;

(3) $D_{e}=A, \alpha_{e}=\operatorname{Id}_{A}$.

Тройка $(A, G, \alpha)$ называется частичной динамической системой.

Ковариантным представлением частичной динамической системы $(A, G, \alpha)$ называется тройка $(\pi, u, H)$, где $\pi: A \rightarrow B(H)$ - представление алгебры $A$ в гильбертовом пространстве $H$ (здесь $B(H)$ - алгебра всех линейных ограниченных операторов в $H), u: G \rightarrow B(H)$ - функция, $u(g)=u_{g}, g \in G$, где $u_{g}$ - частичная изометрия в $H$ с начальным подпространством $\left[\pi\left(D_{g^{-1}}\right) H\right]$ и финальным подпространством $\left[\pi\left(D_{g}\right) H\right]$, такая, что

(1) $u_{g} \pi(d) u_{g^{-1}}=\pi\left(\alpha_{g}(d)\right), d \in D_{g^{-1}}$, 
(2) $\pi(d)\left(u_{g} u_{h}-u_{g h}\right)=0, d \in D_{g} \cap D_{g h}$,

(3) $u_{g}^{*}=u_{g^{-1}}$.

Пусть

$$
L=\left\{a \in l^{1}(G, A): a(g) \in D_{g}\right\}
$$

с обычной нормой $\|a\|_{1}=\sum\|a(g)\|$. Определим (сверточное) умножение и инволюцию на $L$ следующим образом:

$$
(a * b)(g)=\sum_{h \in G} \alpha_{h}\left(\alpha_{h^{-1}}(a(h)) b\left(h^{-1} g\right)\right), \quad a^{*}(g)=\alpha_{g}\left(a\left(g^{-1}\right)^{*}\right) .
$$

С этими операциями $L$ становится банаховой *-алгеброй.

ОПРЕДЕЛЕНИЕ. Скрещенное произведение, ассоциированное с частичной динамической системой $(A, G, \alpha)$, - это обертывающая $C^{*}$-алгебра для $L$, обозначаемая через $A \times{ }_{\alpha} G$.

Пусть $(\pi, u, H)$ - ковариантное представление системы $(A, G, \alpha)$. Зададим представление $\pi \times u: L \rightarrow B(H)$ формулой

$$
(\pi \times u)(a)=\sum \pi(a(g)) u_{g} .
$$

Из определения скрещенного произведения $A \times_{\alpha} G$ следует, что $\pi \times u$ продолжается до *-представления этого произведения.

Приведенное частичное скрещенное произведение. Среди ковариантных представлений системы $(A, G, \alpha)$ одно из важнейших - так называемое приведенное скрещенное произведение, которое определяется следующим образом (см. [2, Sec. 3]).

Прежде всего, по каждому представлению $\pi: A \rightarrow B(H)$ мы строим представление $\tilde{\pi}$ (так называемое регулярное представление) алгебры $A$ в $l^{2}(G, H)$. Пусть $\pi_{g}: D_{g} \rightarrow B(H)$ задается формулой $\pi_{g}(d)=\pi\left(\alpha_{g^{-1}}(d)\right)$. Согласно п. 2.10.4 из [4], существует единственное продолжение $\pi_{g}^{\prime}$ представления $\pi_{g}$ на $A$, аннулирующее $\left[\pi_{g}\left(D_{g}\right) H\right]^{\perp}$. Оно имеет вид

$$
\pi_{g}^{\prime}(d)=s \lim _{\gamma} \pi_{g}\left(v_{\gamma} d\right)
$$

где $\left\{v_{\gamma}\right\}_{\gamma}$ - аппроксимативная единица для $D_{g}$. Теперь мы определяем представление $\tilde{\pi}: A \rightarrow B\left(l^{2}(G, H)\right)$ формулой

$$
\tilde{\pi}(d) \xi(g)=\pi_{g}^{\prime}(d) \xi(g), \quad \xi \in l^{2}(G, H), g \in G, d \in A .
$$

Для регулярного представления $\lambda$ группы $G, \lambda: G \rightarrow B\left(l^{2}(G, H)\right),\left(\lambda_{g} \xi\right)(h)=$ $\xi\left(g^{-1} h\right)$, имеем [2, Proposition 3.1]

$$
\lambda_{g} \tilde{\pi}(d) \lambda_{g^{-1}}=\tilde{\pi}\left(\alpha_{g}(d)\right), \quad g \in G, d \in D_{g^{-1}}
$$

Если мы положим $\tilde{\lambda}_{g}=\lambda_{g} P_{g^{-1}}$, где $P_{g}$ - ортогональный проектор на гильбертово пространство $\left[\tilde{\pi}\left(D_{g}\right) l^{2}(G, H)\right]$, то $\left(\tilde{\pi}, \tilde{\lambda}, l^{2}(G, H)\right)$ станет ковариантным представлением системы $(A, G, \alpha)$.

Пусть $\|\cdot\|_{r}-$ норма в $l^{1}(G, A)$ вида $\|a\|_{r}=\sup \{\|(\tilde{\pi} \times \lambda)(a)\|(\pi, H) \in \operatorname{Rep}(A)\}$, где $\operatorname{Rep}(A)$ - множество всех представлений алгебры $A$.

Приведенное скрещенное произведение $A \times_{\alpha r} G$, ассоциированное с $(A, G, \alpha)$, - это пополнение пространства $l^{1}(G, A)$ относительно нормы $\|\cdot\|_{r}$. 
В действительности, для того чтобы задать приведенное скрещенное произведение, не обязательно использовать все представления алгебры $A$. Как показывает следующий результат, достаточно любого ее точного представления.

Tеорема 1.1 [2, Proposition 3.4]. Пусть $\pi: A \rightarrow B(H)$ - представление. Тогда $\tilde{\pi}$ является точным в том и только том случае, когда $\tilde{\pi} \times \lambda$ является точным представлением алгебры $A \times_{\alpha r} G$.

\section{§2. Свойство $(*)$ и топологически свободное частичное действие}

ОПРЕДЕЛЕНИЕ. Пусть $(\pi, u, H)$ - ковариантное представление системы $(A, G, \alpha)$. Будем говорить, что $\pi \times u$ обладает свойством $(*)$, если для любой конечной суммы $\sum \pi(a(g)) u_{g}$ выполняется неравенство $\left\|\sum \pi(a(g)) u_{g}\right\| \geqslant\|a(e)\|$.

ЗАмечание 2.1. Из [2, Proposition 3.5] следует, что $A \times_{\alpha r} G$ и $A \times_{\alpha} G$ обладают свойством $(*)$.

Если $\pi \times u$ обладает свойством $(*)$, то определенное на конечных суммах отображение $\mathscr{N}\left(\sum_{g \in F} \pi(a(g)) u_{g}\right)=a(e)$ единственным образом продолжается до (положительного, не увеличивающего норму условного ожидания) отображения $\mathscr{N}:(\pi \times u)\left(A \times{ }_{\alpha} G\right) \rightarrow A$.

Переходим к одному из основных понятий статьи - топологически свободному частичному действию.

Для любого идеала $J \subset A$ мы полагаем supp $J=\{x \in \operatorname{Prim} A: x \not \supset J\}$. Известно [4, 3.2.1], что отображение $x \rightarrow x \cap J$ устанавливает гомеоморфизм $\operatorname{supp} J \leftrightarrow \operatorname{Prim} J$ (относительно топологии Джекобсона) и $\operatorname{supp} J$ является открытым множеством в $\operatorname{Prim} A$. Положим также $\widehat{A}^{J}=\{\pi \in \widehat{A}: \pi(J) \neq 0\}$ (здесь $\widehat{A}$ - спектр алгебры $A)$. Отображение $\left.\pi \rightarrow \pi\right|_{J}$ устанавливает гомеоморфизм $\widehat{A}^{J} \leftrightarrow \widehat{J}$ (относительно топологии Джекобсона), и $\widehat{A}^{J}$ - открытое множество в $\widehat{A}[4,3.2 .1]$.

ОПредЕЛЕНиЕ. Зададим отображение $\tau_{g}: \widehat{A}^{D_{g-1}} \rightarrow \widehat{A}^{D_{g}}$ следующим образом: для каждого $\pi \in \widehat{A}^{D_{g-1}}$ полагаем $\tau_{g}(\pi)(j)=\pi\left(\alpha_{g}^{-1}(j)\right), j \in D_{g}$. Из приведенных выше наблюдений следует, что $\tau_{g}$ - гомеоморфизм.

Зададим также отображение $t_{g}: \operatorname{supp} D_{g-1} \rightarrow \operatorname{supp} D_{g}$ по следующему правилу: для каждой точки $x \in \operatorname{supp} D_{g^{-1}}$, такой, что $x=\operatorname{ker} \pi$, где $\pi \in \widehat{A}^{D_{g^{-1}}}$, полагаем $t_{g}(x)=\operatorname{ker} \tau_{g}(\pi)$. Ясно, что $t_{g}$ - гомеоморфизм.

Тогда $\left\{\tau_{g}\right\}_{g \in G}$ определяет частичное действие группы $G$ частичными гомеоморфизмами на $\widehat{A}$, а $\left\{t_{g}\right\}_{g \in G}$ определяет частичное действие группы $G$ частичными гомеоморфизмами на $\operatorname{Prim} A$.

ОПРЕДЕЛЕНИЕ. Будем говорить, что частичное действие $\left\{\alpha_{g}\right\}_{g \in G}$ mоnологически свободно, если для каждого конечного множества $\left\{g_{1}, \ldots, g_{k}\right\} \subset G$ и любого непустого открытого множества $U \subset \operatorname{supp} D_{g_{1}^{-1}} \cap \cdots \cap \operatorname{supp} D_{g_{k}^{-1}}$ существует точка $x \in U$, такая, что все точки $t_{g_{i}}(x), i=1, \ldots, k$, различны.

Это условие можно также сформулировать следующим образом: для каждого конечного множества $\left\{g_{1}, \ldots, g_{k}\right\} \subset G$ и любого непустого открытого множества $U$ существует точка $x \in U$, такая, что все точки $t_{g_{i}}(x), i=1, \ldots, k$, которые определены (т. е. $\left.x \in \operatorname{supp} D_{g_{i}^{-1}}\right)$, различны. 
Если для каждого $g \in G$ мы введем в рассмотрение множество $X_{g}=\{x \in$ supp $\left.D_{g^{-1}}: t_{g}(x)=x\right\}$, то упомянутое выше условие может быть переписано так: для любого конечного множества $\left\{g_{1}, \ldots, g_{n}\right\}, g_{i} \neq e$, внутренность множества $\bigcup_{i=1}^{n} X_{g_{i}}$ пуста.

Основным утверждением данного параграфа является теорема 2.4, а техническим результатом - лемма 2.3. Одним из технических инструментов доказательства этой леммы служит следующая лемма 2.2, которая полезная и сама по себе.

Лемма 2.2 [5, Lemma 12.15]. Пусть $D$ есть $C^{*}$-подалгебра алгебрь $B(H)$ линейных ограниченных операторов в гильбертовом пространстве $H$ и $D^{\prime}-$ коммутант алгебры $D$. Если $P_{1}, P_{2} \in D^{\prime}-$ два ортогональных проектора, таких, что сужения $\left.D\right|_{H_{P_{1}}}$ u $\left.D\right|_{H_{P_{2}}}\left(\right.$ где $\left.H_{P_{1}}=P_{1}(H), H_{P_{2}}=P_{2}(H)\right)$ являются неприводимыми и различными представлениями, то $H_{P_{1}} \perp H_{P_{2}}$.

Лемма 2.3. Пусть частичное действие $\left\{\alpha_{g}\right\}_{g \in G}$ топологически свободно $u \pi \times u$ - представление, такое, что $\pi$ является точным представлением алгебры $A$. Пусть $F-$ конечное подмножество в $G, a \in L-$ любая функиия, такая, что $a(g)=0, g \notin F, u c \in(\pi \times u)(L)$ - оператор вида

$$
c=\sum_{g \in F} \pi(a(g)) u_{g} .
$$

Тогда для любого $\varepsilon>0$ существует неприводимое представление $\pi^{\prime}$ алгебрь $\pi(A)$, такое, что для любого неприводимого представления $\nu$ алгебры $(\pi \times u)\left(A \times{ }_{\alpha} G\right)$, являющегося продолэсением представления $\pi^{\prime}$, выполняются условия

(i) $\left\|\pi^{\prime}(\pi(a(e)))\right\| \geqslant\|a(e)\|-\varepsilon$,

(ii) $P_{\pi^{\prime}} \pi^{\prime}(\pi(a(e))) P_{\pi^{\prime}}=P_{\pi^{\prime}} \nu(c) P_{\pi^{\prime}}$, где $P_{\pi^{\prime}}$ - ортогональный проектор на $H_{\pi^{\prime}}$ в $H_{\nu}$, а $H_{\pi^{\prime}}$ и $H_{\nu}$ - пространства представлений $\pi^{\prime}$ и $\nu$ соответственно.

ДокАЗАтЕльство. Так как $\pi(A) \cong A$, то в дальнейшем мы будем их отождествлять.

Для любого $d \in A$ и $x \in \operatorname{Prim} A$ через $\breve{d}(x)$ обозначается число $\inf _{j \in x}\|d+j\|$. Для каждого $d \in A$ функция $\breve{d}(x)$ полунепрерывна снизу на $\operatorname{Prim} A$ и достигает своей верхней грани, равной $\|d\|[4,3.3 .2,3.3 .6]$.

Пусть $x_{0} \in \operatorname{Prim} A$ - точка, в которой $\breve{a}(e)\left(x_{0}\right)=\|a(e)\|$, и $\pi_{0}$ - неприводимое представление алгебры $A$, такое, что $x_{0}=\operatorname{ker} \pi_{0}$ (т. е. $\left.\left\|\pi_{0}(a(e))\right\|=\|a(e)\|\right)$. Taк как функция $\breve{a}(e)(x)$ полунепрерывна снизу, то для любого $\varepsilon>0$ существует открытое множество $U \subset \operatorname{Prim} A$, такое, что

$$
\breve{a}(e)(x)>\|a(e)\|-\varepsilon \quad \text { для каждого } x \in U .
$$

Из топологической свободы частичного действия $\left\{\alpha_{g}\right\}_{g \in G}$ вытекает существование точки $x^{\prime} \in U$, такой, что все точки $t_{g_{i}}\left(x^{\prime}\right), i=1, \ldots, k$, различны (если они определены, т. е. $\left.x^{\prime} \in \operatorname{supp} D_{g_{i}^{-1}}\right)$.

Пусть $\pi^{\prime}$ - неприводимое представление алгебры $A$, такое, что $\operatorname{ker} \pi^{\prime}=x^{\prime}$, и пусть $\nu$ - произвольное продолжение представления $\pi^{\prime}$ до неприводимого представления алгебры $(\pi \times u)\left(A \times{ }_{\alpha} G\right)$. Мы будем обозначать той же буквой $\nu$ продолжение упомянутого представления до неприводимого представления $C^{*}$-алгебры $C$, порожденной алгеброй $(\pi \times u)\left(A \times_{\alpha} G\right)$ и элементами $\left\{u_{g}\right\}_{g \in G}$ (см. $[4,2.10 .2])$. Для этого представления $\nu$ имеет место включение $H_{\pi^{\prime}} \subset H_{\nu}$. 
Из выбора представления $\pi^{\prime}$ и неравенства (2.2) мы заключаем, что существует вектор $\xi \in H_{\pi^{\prime}}$, такой, что $\|\xi\|=1$ и $\left\|\pi^{\prime}(a(e)) \xi\right\|>\|a(e)\|-\varepsilon$. Итак, п. (i) доказан.

Для доказательства п. (ii) установим, что для любых векторов $\xi, \eta \in H_{\pi^{\prime}}$

$$
\left\langle\pi^{\prime}\left(d_{1}\right) \eta, \nu\left(d_{2} u_{g}\right) \xi\right\rangle=0, \quad d_{1} \in A, d_{2} \in D_{g}, g \in F, g \neq e .
$$

Из этого, в свою очередь, следует, что

$$
P_{\pi^{\prime}} \nu\left(d_{2} u_{g}\right) P_{\pi^{\prime}}=0, \quad g \in F, d_{2} \in D_{g}, g \neq e .
$$

Чтобы проверить равенство (2.3), рассмотрим следующие положения точки $x^{\prime}$ :

1) $x^{\prime} \notin \operatorname{supp} D_{g}$. В этом случае $\pi^{\prime}\left(d_{2}^{*}\right)=0$ и

$$
\left\langle\pi^{\prime}\left(d_{1}\right) \eta, \nu\left(d_{2}\right) \nu\left(u_{g}\right) \xi\right\rangle=\left\langle\nu\left(d_{2}^{*}\right) \pi^{\prime}\left(d_{1}\right) \eta, \nu\left(u_{g}\right) \xi\right\rangle=\left\langle\pi^{\prime}\left(d_{2}^{*}\right) \pi^{\prime}\left(d_{1}\right) \eta, \nu\left(u_{g}\right) \xi\right\rangle=0 .
$$

2) $x^{\prime} \notin \operatorname{supp} D_{g^{-1}}$. Замечая, что $\nu\left(u_{g}^{*} u_{g}\right)$ - проектор на существенное пространство для $\nu\left(D_{g^{-1}}\right)$, мы заключаем, что $\nu\left(u_{g}^{*} u_{g}\right) \xi=0$ и поэтому

$$
\begin{aligned}
\left\langle\pi^{\prime}\left(d_{1}\right) \eta, \nu\left(d_{2}\right) \nu\left(u_{g}\right) \xi\right\rangle & =\left\langle\pi^{\prime}\left(d_{1}\right) \eta, \nu\left(d_{2}\right) \nu\left(u_{g} u_{g}^{*} u_{g}\right) \xi\right\rangle \\
& =\left\langle\pi^{\prime}\left(d_{1}\right) \eta, \nu\left(d_{2}\right) \nu\left(u_{g}\right) \nu\left(u_{g}^{*} u_{g}\right) \xi\right\rangle=0 .
\end{aligned}
$$

3) Наконец, пусть $x^{\prime} \in \operatorname{supp} D_{g} \cap \operatorname{supp} D_{g^{-1}}$. В этом случае $\pi^{\prime}-$ неприводимое представление как для $D_{g}$, так и для $D_{g^{-1}}$, и $t_{g}\left(x^{\prime}\right) \in \operatorname{supp} D_{g}$ (в соответствии с определением $t_{g}$ в начале параграфа). Более того, мы имеем

$$
\nu\left(u_{g}^{*} u_{g}\right) \eta=\eta, \quad \nu\left(u_{g} u_{g}^{*}\right) \eta=\eta \quad \text { для любого } \eta \in H_{\pi^{\prime}} .
$$

Так как $\nu\left(u_{g}\right)$ - частичная изометрия, то из формул $(2.5)$ следует, что $H_{\pi^{\prime}}$ принадлежит как начальному, так и финальному подпространствам частичных изометрий $\nu\left(u_{g}\right)$, а также начальному и финальному подпространствам для $\nu\left(u_{g}^{*}\right)$ и отображения $\nu\left(u_{g}\right): H_{\pi^{\prime}} \rightarrow \nu\left(u_{g}\right)\left(H_{\pi^{\prime}}\right)$ и $\nu\left(u_{g}^{*}\right): H_{\pi^{\prime}} \rightarrow \nu\left(u_{g}^{*}\right)\left(H_{\pi^{\prime}}\right)$ являются изоморфизмами.

Пусть $P_{1}$ - ортогональный проектор в $H_{\nu}$ на $H_{\pi^{\prime}}$. Согласно определению представления $\nu$, мы имеем $P_{1} \in \nu(A)^{\prime}$. Равенства (2.5) означают, что

$$
P_{1}=P_{1} \nu\left(u_{g}^{*} u_{g}\right)=P_{1} \nu\left(u_{g} u_{g}^{*}\right) .
$$

Положим $P_{2}=\nu\left(u_{g}\right) P_{1} \nu\left(u_{g}^{*}\right)$. Из приведенных выше наблюдений следует, что $\nu\left(u_{g}\right): P_{1}\left(H_{\pi^{\prime}}\right) \rightarrow P_{2}\left(H_{\pi^{\prime}}\right)$ является изоморфизмом. Заметим также, что

$$
P_{2} \in\left(\nu\left(D_{g}\right)\right)^{\prime} .
$$

Действительно, для любого $d \in D_{g}$ имеем

$$
\begin{gathered}
\nu(d)=\nu\left(u_{g} u_{g}^{*}\right) \nu(d)=\nu(d) \nu\left(u_{g} u_{g}^{*}\right), \\
\nu\left(\alpha_{g^{-1}}(d)\right)=\nu\left(u_{g}^{*} u_{g}\right) \nu\left(\alpha_{g^{-1}}(d)\right)=\nu\left(\alpha_{g^{-1}}(d)\right) \nu\left(u_{g}^{*} u_{g}\right), \\
\nu\left(u_{g}^{*}\right) \nu(d) \nu\left(u_{g}\right)=\nu\left(\alpha_{g^{-1}}(d)\right), \quad \nu\left(\alpha_{g} \circ \alpha_{g^{-1}}(d)\right)=\nu(a) .
\end{gathered}
$$

Пользуясь этими равенствами, мы для каждого $d \in D_{g}$ получаем $P_{2} \nu(d)=$ $\nu(d) P_{2}$.

Кроме того, неприводимость представления $\left.\nu\left(D_{g}\right)\right|_{H_{P_{1}}}$ влечет за собой неприводимость представления $\left.\nu\left(D_{g}\right)\right|_{H_{P_{2}}}$ (здесь $H_{P_{1}}=P_{1}\left(H_{\nu}\right)=H_{\pi^{\prime}}$ и $H_{P_{2}}=P_{2}\left(H_{\nu}\right)$ ). 
Заметим теперь, что для $d \in D_{g}$

$$
P_{1} \nu(d)=0 \Longleftrightarrow \breve{d}\left(x^{\prime}\right)=0, \quad P_{2} \nu(d)=0 \Longleftrightarrow \breve{d}\left(t_{g}\left(x^{\prime}\right)\right)=0 .
$$

Таким образом (так как точки $x^{\prime}$ и $t_{g}\left(x^{\prime}\right)$ различны), мы заключаем, что представления $\left.\nu\left(D_{g}\right)\right|_{H_{P_{1}}}$ и $\left.\nu\left(D_{g}\right)\right|_{H_{P_{2}}}$ различны. Применяя лемму 2.2 , мы получаем

$$
P_{1} \cdot P_{2}=0 \text {. }
$$

Из формул (2.8), (2.5), (2.6) и (2.7) следует (2.3).

Возвращаясь теперь к оператору (2.1) (напомним, что мы отождествляем $A$ и $\pi(A))$ и используя равенство $(2.4)$, мы получаем

$$
P_{\pi^{\prime}} \nu\left(\sum_{g \in F} a(g) u_{g}\right) P_{\pi^{\prime}}=P_{\pi^{\prime}} \nu(a(e)) P_{\pi^{\prime}}=P_{\pi^{\prime}} \pi^{\prime}(a(e)) P_{\pi^{\prime}} ;
$$

таким образом, условие (ii) выполняется, и доказательство леммы закончено.

ТЕорема 2.4. Пусть частичное действие $\left\{\alpha_{g}\right\}_{g \in G}$ топологически свободно. Если представление $\pi \times$ и таково, что $\pi$ является точным представлением алгебры $A$, то $\pi \times и$ обладает свойством (*).

ДокАзАтЕльство. Пусть $c$ - оператор вида (2.1). Возьмем $\pi^{\prime}$, упомянутое в утверждении леммы 2.3. Тогда из (ii) и (i) вытекает, что

$$
\|c\| \geqslant\|\nu(c)\| \geqslant\left\|P_{\pi^{\prime}} \nu(c) P_{\pi^{\prime}}\right\| \geqslant\|a(e)\|-\varepsilon .
$$

Поскольку $\varepsilon$ произвольно, отсюда следует $(*)$.

ЛЕмма 2.5. Пусть частичное действие $\left\{\alpha_{g}\right\}_{g \in G}$ топологически свободно $u$ представление $\pi \times u$ таково, что $\pi$ - точное представление алгебры А. Тогда для любого $c \in(\pi \times u)\left(A \times{ }_{\alpha} G\right)$ и любого $\varepsilon>0$ существует неприводимое представление $\pi^{\prime}$ алгебры $\pi(A)$, такое, что для любого неприводимого представления $\nu$ алгебры $(\pi \times u)\left(A \times{ }_{\alpha} G\right)$, являющегося продолэсением $\pi^{\prime}$,

(i) $\left\|\pi^{\prime}(\mathscr{N}(c))\right\| \geqslant\|\mathscr{N}(c)\|-\varepsilon$,

(ii) $\left\|P_{\pi^{\prime}} \pi^{\prime}(\mathscr{N}(c)) P_{\pi^{\prime}}-P_{\pi^{\prime}} \nu(c) P_{\pi^{\prime}}\right\| \leqslant \varepsilon$.

ДокАЗАТЕЛЬСтво получается стандартным аппроксимационным рассуждением ввиду плотности конечных сумм вида $(2.1)$ в $(\pi \times u)\left(A \times{ }_{\alpha} G\right)$ и того факта, что $\pi \times u$ обладает свойством $(*)$.

\section{§3. Скрещенные произведения и приведенные скрещенные произведения}

Скрещенное произведение $A \times{ }_{\alpha} G$ естественно рассматривать как максимальную $C^{*}$-алгебру, обладающую свойством $(*)$ (это следует из конструкции $A \times{ }_{\alpha} G$ и замечания 2.1). С другой стороны, Эксель показал, что приведенное скрещенное произведение $A \times_{\alpha r} G$ является минимальной $C^{*}$-алгеброй, обладающей этим свойством. Точное значение «минимальности» дается в следующем утверждении, которое является переформулировкой для рассматриваемой ситуации теоремы 3.3 из [6] (напомним при этом, что, согласно теореме 1.1 настоящей статьи, $\tilde{\pi} \times \lambda$ для любого точного представления $\pi$ алгебры $A$ является точным представлением алгебры $\left.A \times_{\alpha r} G\right)$. 
Теорема 3.1. Пусть $\pi \times u-$ представление, для которого $\pi$ - точное представление алгебры А. Если $\pi \times$ и обладает свойством (*), то отображение

$$
(\pi \times u)\left(A \times_{\alpha} G\right) \ni \sum \pi(a(g)) u_{g} \mapsto \sum \tilde{\pi}(a(g)) \tilde{\lambda}_{g} \in(\tilde{\pi} \times \lambda)\left(A \times_{\alpha r} G\right)
$$

продолюается до эпиморбизма $C^{*}$-алгебр (здесь $(\tilde{\pi} \times \lambda)-$ представление, упомлнутое в теореме 1.1).

ЗАмечАниЕ 3.2. Известно также, что если $G$ - аменабельная группа, то каноническая сюръекция $\Lambda: A \times_{\alpha} G \rightarrow A \times_{\alpha r} G$ является изоморфизмом (см., например, [2, Proposition 4.2]).

Это замечание вместе с теоремой 3.1 приводит к следующему результату.

Теорема 3.3. Пусть $G$ - аменабельная группа $u\left(\pi^{i}, u^{i}, H^{i}\right), i=1,2,-\partial в а$ ковариантных представления системы $(A, G, \alpha)$, таких, что оба представления $\pi^{i} \times u^{i}, i=1,2$, обладают свойством $(*)$. Тогда отображение

$$
\sum \pi^{1}(a(g)) u_{g}^{1} \mapsto \sum \pi^{2}(a(g)) u_{g}^{2}
$$

задает изоморфизм алгебр $\left(\pi^{1} \times u^{1}\right)\left(A \times{ }_{\alpha} G\right) u\left(\pi^{2} \times u^{2}\right)\left(A \times_{\alpha} G\right)$.

ЗАмечание 3.4. Важность свойства $(*)$ впервые (по-видимому) была выявлена О'Донованом [7] в связи с описанием $C^{*}$-алгебр, порожденных взвешенными сдвигами. Наиболее общий результат (типа теоремы 3.3), устанавливающий принципиальную роль этого свойства в теории скрещенных произведений $C^{*}$-алгебр на дискретные группы автоморфизмов, был получен в [8] для произвольной $C^{*}$-алгебры и аменабельной дискретной группы (см. также $[5$, Chap. 2,3$]$ по поводу полных доказательств и различных приложений). Взаимосвязи соответствующего свойства со скрещенными произведениями на эндоморфизмы, порожденные изометриями, исследовались в $[9,10]$.

Следует отметить, что в [5] теорема 12.8 (аналог нашей теоремы 3.3) доказана прямым способом, не использующим приведенного скрещенного произведения. Таким образом, в частности, изоморфизм $\Lambda: A \times_{\alpha} G \rightarrow A \times_{\alpha r} G$ для аменабельных групп может быть выведен из этого результата (доказательство теоремы 12.8 из [5] легко переносится на ситуацию скрещенного произведения, ассоциированного с частичной динамической системой).

Теорема 3.5. Пусть частичное действие $\left\{\alpha_{g}\right\}_{g \in G}$ топологически свободно и представление $\pi \times$ и таково, что $\pi$ является точным представлением алгебры А. Тогда отображение

$$
(\pi \times u)\left(A \times_{\alpha} G\right) \ni \sum \pi(a(g)) u_{g} \mapsto \sum \tilde{\pi}(a(g)) \tilde{\lambda}_{g} \in(\tilde{\pi} \times \lambda)\left(A \times_{\alpha r} G\right)
$$

продолжается до эпиморфизма $C^{*}$-алгебр (здесь $\tilde{\pi} \times \lambda-$ представление, упомянутое в теореме 1.1).

Для доказательства применяем теорему 2.4 и теорему 3.1.

Теорема 3.6. Пусть $G$ - аменабельная группа и частичное действие $\left\{\alpha_{g}\right\}_{g \in G}$ топологически свободно. Если $\left(\pi^{i}, u^{i}, H^{i}\right), i=1,2,-$ два ковариантных представления для $(A, G, \alpha)$, таких, что оба представления $\pi^{i}, i=1,2$, являются точными представлениями алгебры $A$, то отображение

$$
\sum \pi^{1}(a(g)) u_{g}^{1} \mapsto \sum \pi^{2}(a(g)) u_{g}^{2}
$$

задает изоморбизм алгебр $\left(\pi^{1} \times u^{1}\right)\left(A \times{ }_{\alpha} G\right) u\left(\pi^{2} \times u^{2}\right)\left(A \times_{\alpha} G\right)$. 
Применяем теорему 2.4 и теорему 3.3 .

Следующие теорема 3.7 и следствие 3.8 в некотором смысле противоположны теореме 3.1. Они составляют также обобщение теоремы 2.6 из [11] (где $A=$ $\left.C_{0}(X)\right)$.

ТеОремА 3.7. Пусть частичное действие $\left\{\alpha_{g}\right\}_{g \in G}$ топологически свободно. Если $I-$ идеал в $A \times{ }_{\alpha r} G$, mо $I=\{0\}$ тогда и только тогда, когда $I \cap A=\{0\}$.

Доказательство. Пусть $I \cap A=\{0\}$. Обозначим через $\pi: A \times{ }_{\alpha r} G \rightarrow\left(A \times{ }_{\alpha r}\right.$ $G) / I$ факторотображение, и пусть $c \in I-$ любой элемент, такой, что $c \geqslant 0$ и $\pi(c)=0$. Для доказательства равенства $I=\{0\}$ достаточно проверить, что

$$
c=0 \text {. }
$$

Так как отображение $\mathscr{N}: A \times_{\alpha r} G \rightarrow A$, определенное после замечания 2.1, является точным (см., например, [6, Proposition 2.12]), то равенство (3.1) будет доказано, если мы установим, что

$$
\mathscr{N}(c)=0
$$

Поэтому проверим это равенство.

Из равенства $I \cap A=\{0\}$ вытекает, что $\pi(A) \cong A$. Задавшись произвольным $\varepsilon>0$, возьмем представление $\pi^{\prime}$ из утверждения леммы 2.5 (мы можем считать его представлением как алгебры $\pi(A)$, так и алгебры $A)$ и продолжим его до неприводимого представления $\nu$ алгебры $\pi\left(A \times{ }_{\alpha r} G\right)$ (здесь мы считаем $\pi$ представлением $\pi \times u$ из утверждения леммы 2.5). Очевидно, что $\nu \circ \pi-$ неприводимое представление алгебры $A \times{ }_{\alpha r} G$.

Теперь из условия $\pi(c)=0$ и свойства (ii) в утверждении леммы 2.5 следует, чTо

$$
\varepsilon \geqslant\left\|P_{\pi^{\prime}} \pi^{\prime}(\mathscr{N}(\pi(c))) P_{\pi^{\prime}}-P_{\pi^{\prime}} \nu(\pi(c)) P_{\pi^{\prime}}\right\|=\left\|\pi^{\prime}(\mathscr{N}(\pi(c)))\right\|=\left\|\pi^{\prime}(\mathscr{N}(c))\right\| .
$$

Это вместе со свойством (i) влечет за собой неравенство

$$
\|\mathscr{N}(c)\| \leqslant 2 \varepsilon .
$$

Тем самым (ввиду произвольности $\varepsilon$ ) равенство (3.2) установлено и доказательство завершено.

СлЕДСТВИЕ 3.8. Пусть частичное действие $\left\{\alpha_{g}\right\}_{g \in G}$ топологически свободно. Представление $\pi$ приведенного скрещенного произведения $A \times{ }_{\alpha r} G$ является точным тогда и толъко тогда, когда оно точно на $A$.

ДокАзАТЕльство. Достаточно в утверждении теоремы 3.7 взять $I=\operatorname{ker} \pi$.

ЗАМЕЧАНИЕ 3.9. ВЗаимосвязи между топологической свободой действия и свойством $(*)$, а также приложения этих свойств к различным результатам, связанным со скрещенными произведениями, интенсивно эксплуатировались многими авторами. Начало использования топологической свободы как инструмента исследования идеалов в скрещенных произведениях положено (по-видимому) O'Донованом в [7, Theorem 1.2.1]. Теорема 3.6 в случае коммутативной алгебры $A$ и действия группы $\mathbb{Z}$ автоморфизмами была доказана в $[12,13]$. Развитие этого направления и его многочисленные (не только чисто $C^{*}$-алгебраические) приложения, такие, как, например, конструкция символического исчисления и построение теории разрешимости функционально-дифференциальных уравнений, представлены в $[14,5,15,16]$. Для общей ситуации автоморфизмов теорема 3.6 была получена в [8] (см. также в этой связи [5, Chap. 2, 3]). В [17] 
для $C^{*}$-алгебр с «большим» центром введено понятие топологически свободного действия в терминах сужения действия автоморфизмов на центр и получен соответствующий аналог теоремы 3.6. Среди уже упомянутых «чисто» $C^{*}$-алгебраических источников необходимо отметить фундаментальный вклад в данную тематику, внесенный в [6]. Для случая $A=C_{0}(X)$ в [11] исследован ряд структурных проблем теории скрещенных произведений, ассоциированных с топологически свободным частичным действием.

В случае лебеговых пространств топологической свободе действия соответствует так называемая метрическая свобода. Взаимосвязь между этим свойством, свойством $(*)$ и соответствующими результатами для скрещенных произведений (для случая автоморфизмов) была исследована и применена к решению проблемы классификации сохраняющих меру автоморфизмов Арвесоном и Джозефсоном в $[18,19]$.

В ситуации эндоморфизмов, а именно, в случае, когда эндоморфизм $C^{*}$-алгебры порождается одной изометрией, взаимосвязи между топологической свободой действия и свойством (*) исследованы в $[20,8,21]$, где, в частности, получены аналоги теорем 2.4, 3.3, 3.6 для рассматриваемой ситуации. В действительности это исследование было инспирировано пионерскими работами Арзуманяна и Вершика [22-25], в которых были введены и изучены соответствующие объекты в лебеговых пространствах. В последнее время данная тематика получила новое развитие в работах Экселя [26,27] и Экселя и Вершика [28].

\section{ЛитерАТУРА}

1. Exel R. Circle actions on $C^{*}$-algebras, partial automorphisms and generalized Pimsner-Voiculescu exact sequence. J. Funct. Anal., 122, 361-401 (1994).

2. McClanahan $K$. $K$-theory for partial crossed products by discrete groups. J. Funct. Anal., 130, 77-117 (1995).

3. Paterson Alan L. T. Groupoids, inverse semigroups, and their operator algebras. Progr. Math., Vol. 170, Birkhäuser, 1999.

4. Dixmier J. Les $C^{*}$-algebres et leurs representations. Gauthier-Villars Editeur, 1969.

5. Antonevich A., Lebedev A. Functional differential equations: I. $C^{*}$-theory. Pitman Monographs Surveys Pure Appl. Math., Vol. 70, Longman Scientific \& Technical, Harlow, 1994.

6. Exel R. Amenability for Fell bundles. J. Reine Angew. Math., 492, 41-73 (1997).

7. O'Donovan D. P. Weighted shifts and covariance algebras. Trans. Amer. Math. Soc., 208, 1-25 (1975).

8. Лебедев A. B. О некоторых $C^{*}$-методах, применяемых при исследовании алгебр, ассоциированных с автоморфизмами и эндоморфизмами. Деп. в ВИНИТИ, №5351-B 87, 1987.

9. Boyd S., Keswani N., Raeburn I. Faithful representations of crossed products by endomorphisms. Proc. Amer. Math. Soc., 118, No. 2, 427-436 (1993).

10. Adji S., Laca M., Nilsen M., Raeburn I. Crossed products by semigroups of endomorphisms and the Toeplitz algebras of ordered groups. Proc. Amer. Math. Soc., 122, No. 4, 1133-1141 (1994).

11. Exel R., Laca M., Quigg J. Partial dynamical systems and $C^{*}$-algebras generated by partial isometries. J. Operator Theory, 47, 169-186 (2002).

12. Лебедев A. B. Об обратимости элементов в $C^{*}$-алгебрах, порожденных динамическими системами. УМН, 34, No. 4, 199-200 (1979).

13. Лебедев A. B. Операторы типа взвешенного сдвига. Дис. канд. физ.-мат. наук, Минск, 1980. 
14. Антоневич А. Б. Линейные функциональные уравнения. Операторный подход. Университетское, Минск, 1988.

15. Antonevich A., Belousov M., Lebedev A. Functional differential equations: II. $C^{*}$-applications. Part 1. Equations with continuous coefficients. Pitman Monographs Surveys Pure Appl. Math., Vol. 94, Longman, Harlow, 1998.

16. Antonevich A., Belousov M., Lebedev A. Functional differential equations: II. $C^{*}$-applications. Part 2. Equations with discontinuous coefficients and boundary value problems. Pitman Monographs Surveys Pure Appl. Math., Vol. 95, Longman, Harlow, 1998.

17. Карлович Ю. И. Локально-траекторный метод исследования обратимости в $C^{*}$-алгебрах операторов с дискретными группами сдвигов. ДАН CCCP, 299, №. 3, 546-550 (1988).

18. Arveson $W$. B. Operator algebras and measure preserving automorphisms. Acta Math., 118, 95-109 (1967).

19. Arveson W. B., Josephson K. B. Operator algebras and measure preserving automorphisms. II. J. Funct. Anal., 4, No. 1, 100-134 (1969).

20. Лебедев A. B. О расширении операторных алгебр с помощью изометрических операторов, порождающих эндоморфизмы. УМН, 39, вып. 5, 247-248 (1984).

21. Лебедев A. B. Конструкции и объекты, ассоциированные с $C^{*}$-динамическими системами, порожденными эндоморфизмами. Труды ИМ НАН Беларуси, 1, 133142 (1998).

22. Арзуманян B. А., Вершик A. М. Фактор-представления скрещенного произведения коммутативной $C^{*}$-алгебры и полугруппы ее эндоморфизмов. Докл. АН CCCP, 238, № 3, 513-517 (1978).

23. Арзуманян B. А., Структура и представления инволютивных алгебр, ассоциированных с полугруппами эндоморфизмов: Дис. канд. физ.-мат. наук, Ленинград, 1978.

24. Arzumanian V. A., Vershik A. M. Star algebras associated with endomorphisms. In: Operator Algebras and Group Representations, Proc. Int. Conf. Neptun/Rom., 1980, Vol. I, Monographs Stud. Math., Vol. 17, 1984, pp. 17-27.

25. Арзуманян B. A. Операторные алгебры, ассоциированные с несингулярными эндоморфизмами пространств Лебега. Изв. АН АрмССР, 20, №6, 596-616 (1986).

26. Exel $R$. A new look at the crossed-product of a $C^{*}$-algebra by an endomorphism. arXiv:math.OA/0012084 v1 12 Dec 2000.

27. Exel $R$. Crossed-products by finite index endomorphisms and KMS states. arXiv:math. OA/0105195 v1 24 May 2001.

28. Exel R., Vershik A. $C^{*}$-algebras of irreversible dynamical systems. arXiv:math.OA/ 0203185 v1 19 May 2002.

Белорусский государственный университет, Минск

University of Bialystok, Bialystok

Поступила в редакцию e-mail: lebedev@bsu.by 